\title{
Multidimensional Models for Methodological Validation in Multifractal Analysis
}

\author{
R. Lopes $^{a}$, I. Bhouri ${ }^{b}$, S. Maouche ${ }^{c}$, P. Dubois ${ }^{a}$, M.H. Bedoui ${ }^{d}$ and N. Betrouni $^{a}{ }^{1}$ \\ ${ }^{a}$ Inserm, U703, THIAIS, Pavillon Vancostenobel, CHU Lille, France \\ ${ }^{b}$ Université des Sciences et Technologies de Lille, CNRS UMR 8146 Laboratoire LAGIS, \\ Villeneuve d'Ascq, France \\ ${ }^{c}$ Faculté des Sciences de Monastir, Unitde recherche Ondelettes et Multifractals, TIM, Tunisie \\ ${ }^{d}$ Faculté de Médecine de Monastir, équipe Technologie et Imagerie Médicale TIM-99UR-08-27, \\ Tunisie
}

\begin{abstract}
Multifractal analysis is known as a useful tool in signal analysis. However, the methods are often used without methodological validation. In this study, we present multidimensional models in order to validate multifractal analysis methods.
\end{abstract}

Key words: multifractal analysis, models, validation

AMS subject classification: 28A80, 60G18

\section{Introduction}

Multifractal analysis initially appeared with multiplicatives cascades models of Mandelbrot for the study of energy dissipitaion in the context of the fully developed turbulence then it was applied for the measurement of the turbulent flow velocity in the 80's. A signal treatment approach has been developed which consisted to study the regularity of a signal with a velocity v.The aim is to define in each signal point $\mathrm{x}_{0}$ the velocity variation law to deduce the punctual Hölder exponent

${ }^{1}$ Corresponding author. E-mail: r-lopes@chru-lille.fr 
$\mathrm{h}\left(\mathrm{x}_{0}\right)$. Afterwards points with the same exponent $\mathrm{h}$ are grouped together in sets $\mathrm{S}_{h}$. These sets might have a nulle Lebesgue measure. However if they are not significant in measure term, their topological dimension can be. Thus, physicists searched to compute the Hausdorff dimension $\mathrm{d}(\mathrm{h})$ of $S_{h}$. The function $\mathrm{h} \rightarrow \mathrm{d}(\mathrm{h})$ was called the Hausdorff spectrum. By the assumption that $\mathrm{d}(\mathrm{h})$ is $-\infty$ if $S_{h}$ is empty a multifractal function is a function for which $d(h)$ is finite for two different $h$ at least. Riemann's function, Jordan's function and Levy's function for instance are multifractal functions.

The direct determination by numerical computing of the Hausdorff spectrum of a real signal is difficult to estimate because the main inconveniences with such a definition is that it is very difficult to explicitly assign a Hölder exponent to each point, to decompose the signal into its fractal manifolds and, in addition, even when the multifractal decomposition of the signal is available there are numerical problems to provide a good estimate of the fractal dimensions. A formula, named "multifractal formalism" has been established by Parisi and Frisch in order to compute this spectrum. Indeed this propriety proves the equality between the Hausdorff spectrum and another spectrum, called the Legendre spectrum. This formula still to be difficult to apply for real signals, for this reason the topic of many works was the implementation of a method to evaluate the generalized multifractal dimensions spectrum $\mathrm{D}_{q}, \mathrm{q} \in \mathbb{R}$. An alternative description is given by the singularity spectrum $\alpha \rightarrow \mathrm{f}(\alpha)$, where $\alpha$ is the Hölder coefficient [7] and $\mathrm{f}$ is the multifractal spectrum.

Today fractal and multifractal analysis provides powerful tools for physical signals analysis. To deal only with the biomedical field, it covers a vast field from one dimensional signals (electrophysiological signals), to 2D (medical imaging) and 3D (volume reconstruction from tomographic data). Various methods are often applied with the purpose of classification (normal/abnormal, healthy/pathological): their quantitative results are then used in a relative way in order to optimize their discriminative abilities. Furthermore, their use does not always go with a methodological validation, while used methods are approximate value of the theoretical fractal dimension or the theoretical multifractal spectrum, as we mentioned it above. It is interesting to know the precision of methods for the calculation of the approximate multifractal spectrum, i.e. to compare the approximate multifractal spectrum and the theoretical multifractal spectrum. Consequently, the aim of this paper is to present 1D, 2D and 3D models, built according to data of the literature and chosen to reflect the characteristics of the real data. These models are then used as "benchmarks testing". The choice of characteristic for the validation is the multifractal spectrum $\alpha \rightarrow \mathrm{f}(\alpha)$. We chose this representation, because the Hölder coefficient is not sufficient in some cases. Indeed sometimes the Hölder function is very simple, whereas the signal is very irregular (for instance for the fractional Brownian motion, where the Hölder function is constant). Moreover there are signals where the Hölder function is more irregular than the signal (for example the graph of some Iterated function schemes with overlaps). In these cases it's more interesting to use the multifractal spectrum. The obtained results confront the respective theoretical multifractal spectra of these models with those obtained by several methods for multifractal analysis.

The paper is organised as follow: next section is devoted to introduce general aspects of multifractal theory, required for a proper understanding of the methods. Then the models are described in the section 3, we present models in $1 \mathrm{D}, 2 \mathrm{D}$ and $3 \mathrm{D}$. For each model, the theoretical multifractal 
spectrum is presented. The methods are presented in section 4 , we used different multifractal measures and the results of these measures applied of the models are presented in the Results section. The conclusion is finally given in section 6 .

\section{Multifractal theory}

There are lots of works, which explain definitions of various multifractal spectra $[2,9,21]$. We recall the basic definitions and results for a multifractal measure (used in the rest of the manuscript), knowing that the multifractal formalism is different for a multifractal function.

We call multifractal formalism, the concepts which correspond to the singularity spectrum estimation of a singular measure $\mu$. This formalism allows statistically quantifying the regularity fluctuations of an irregular object by continuous spectra of exponents (such as the singularity spectrum). It is defined as follows:

- For all $\mathrm{q} \in \mathbb{R}$, the limit exists:

$$
\tau(q)=\lim _{\delta \rightarrow 0} \frac{\sum_{i} \mu_{i}(\delta)^{q}}{\log (\delta)} .
$$

Where tau(q) is called the partition function and $\delta$ is the neighbour definition.

- The singularity spectrum of $\alpha$ is obtained by Legendre transform of tau:

$$
f(\alpha)=\max _{q \in \mathbb{R}}(\alpha q-\tau(q)) .
$$

A measure is monofractal, if the singularity spectrum is degenerate and limited to one point, i.e. the singularity exponent $\alpha$ is always equal. Thus the partition function of monofractal measure is linear. However a multifractal measure has a singularity spectrum at wide support [19], i.e. there is a continuum of possible singularity exponent.

The spectrum of the equation (2.2) provides a local and global description of a signal. The first description is provided by the Hölder coefficient $\alpha$ [7], and the second description by the multifractal spectrum $\mathrm{f}(\alpha)$, which characterizes the geometrical and statistical repartition of singularities on the signal's support. We can quote that the generalized dimensions $\mathrm{D}_{q}$, introduces in the chaotic dynamical systems theory, are linked at the partition function by the equation:

$$
\tau(q)=\frac{D_{q}}{q-1}
$$

For a multifractal pattern, the $\mathrm{D}_{q}$ dimensions are decreasing functions of $\mathrm{q}$ and $\mathrm{f}(\alpha)$ is a convex function with a maximal value corresponding to the Hausdorff dimension $\mathrm{D}_{H}$. 


\section{Models Generating}

\subsection{D Model}

We analyzed an analytically solvable example (binomial multiplicative process) [18]. Populations or distributions generated by a multiplicative process have many applications and with the advantage that many properties of their distributions may be easily analyzed (self-similar model).

Let's consider the following multiplicative process, which is generated by dividing the unit interval into two pieces, each of half the previous length, but with unequal measure (say $\mathrm{p}_{1}$ and $\mathrm{p}_{2}=1-\mathrm{p}_{1}$ ) and infinitely repeating this process.

Then the measure at the $\mathrm{n}$-th level of this multiplicative process would consist of $\mathrm{N}=2^{n}$ intervals of equal length $\mathrm{L}=2^{-n}$ with probabilities $\mathrm{P}_{k}(\mathrm{~L})=\mathrm{p}_{1}{ }^{n-k} \cdot \mathrm{p}_{2}{ }^{k}, \mathrm{k}=0, \ldots, \mathrm{n}$. The above process is defined by it's partition function with q real:

$$
\begin{gathered}
\tau(q)=\frac{-\log \left(p_{1}^{q}+p_{2}^{q}\right)}{\log (2)} . \\
\alpha(q)=\tau^{\prime}(q)=\frac{d \tau(q)}{d q}=-\frac{p_{1}^{q} \log \left(p_{1}\right)+p_{2}^{q} \log \left(p_{2}\right)}{\left(p_{1}^{q}+p_{2}^{q}\right) \log (2)} .
\end{gathered}
$$

Thus the multifractal spectrum is defined as:

$$
f(q)=q \alpha(q)-\tau(q)=\frac{-q p_{1}^{q} \log \left(p_{1}\right)-q p_{2}^{q} \log \left(p_{2}\right)+\left(p_{1}^{q}+p_{2}^{q}\right) \log \left(p_{1}^{q}+p_{2}^{q}\right)}{\left(p_{1}^{q}+p_{2}^{q}\right) \log (2)} .
$$

Where $\alpha_{q}$ represents the derivative of tau in q defined in equation (3.2) and $\alpha$ is a real. The limit coefficients $\alpha_{\min }$ and $\alpha_{\max }$ are deduced from equation (3.2) and:

$$
\begin{aligned}
& \alpha_{\text {min }}=\alpha(+\infty)=\lim _{q \rightarrow+\infty} \alpha_{q}=\min _{i} \frac{-\log \left(p_{i}\right)}{\log (2)} . \\
& \alpha_{\max }=\alpha(-\infty)=\lim _{q \rightarrow-\infty} \alpha_{q}=\max _{i} \frac{-\log \left(p_{i}\right)}{\log (2)} .
\end{aligned}
$$

\subsection{D Model}

We simulate multifractal singular measurements by using multiplicative cascades models $[18,14]$. A simple model is the p-model, which was introduced in fully developed turbulence [15]. It concerns a multiplicative process of conservative cascades, to model statistical scale-invariance properties of dissipation field in a turbulent flow [16]. Its multifractal properties are well known analytically.

The principle is as follows: we start with one square defined on the interval $[0,1]^{2}$, then we divide the square into four under squares and we assign randomly to each of them a probability $\mathrm{p}_{i}$, such as:

$$
\sum_{i} p_{i}=1
$$




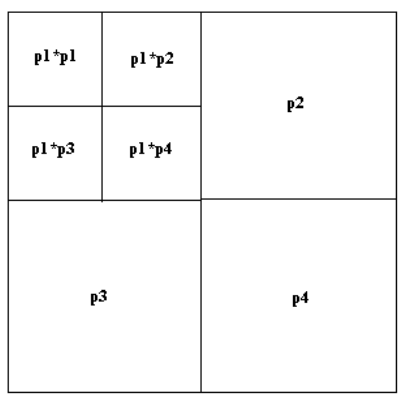

Figure 1: 2D p-model.

We iterate this operation on each under square (Figure 1). The obtained model is thus self-similar, since the square is divided into four equal parts. The $\tau(\mathrm{q})$ function is easily estimated as:

$$
\tau_{q}=\frac{\log \left(p_{1}^{q}+p_{2}^{q}+p_{3}^{q}+p_{4}^{q}\right)}{\log (4)} .
$$

From this, we deduce the $\alpha(\mathrm{q})$ definition:

$$
\alpha_{q}=\frac{d \tau(q)}{d q}=-\frac{\sum_{i=1}^{4} p_{i}^{q} \log \left(p_{i}\right)}{\log (4) \sum_{i=1}^{4} p_{i}^{q}} .
$$

And we obtain $\mathrm{f}(\mathrm{q})$ :

$$
f(q)=q \alpha_{q}-\tau(q)=\frac{-q \sum_{i=1}^{4} p_{i}^{q} \log \left(p_{i}\right)+\log \left(\sum_{i=1}^{4} p_{i}^{q}\right) \sum_{i=1}^{4} p_{i}^{q}}{\log (4) \sum_{i=1}^{4} p_{i}^{q}} .
$$

The $\alpha$ limits were calculated in the same manner as in equations (3.4) and (3.5) by substituing $\log (4)$ by $\log (2)$.

\subsection{D Model}

In 3D, we generated two models, which are the most employed in medical image analysis. Firstly, we extended the 2D model to 3D model, i.e. we construct a self-similar model scaled by a multinomial measure. We started with one cube defined on the interval $[0,1]^{3}$, then we divided the cube into four under cubes and we randomly assigned to each one a probability $\mathrm{p}_{i}$. We iterated the process on each under cubes. Calculations of the theoretical multifractal spectrum are identical to $2 \mathrm{D}$ case, by taking $\mathrm{p}_{i}$, for $\mathrm{i}=1, \ldots, 8$ and by substituting $\log (8)$ to $\log (4)$. 
Secondly we generated another model, which is self-affine. It is an extension of an existing 2D model. We started with one cube defined on the interval $\left[\begin{array}{ll}0 & 1\end{array}\right]^{3}$, we divided it according to three directions, we assigned to some boxes a probability $\mathrm{p}_{i}$ (and $\sum p_{i}=1$ ) and in each one of these boxes we iterated the process. Figure 2 depicts the construction in the $2 \mathrm{D}$.

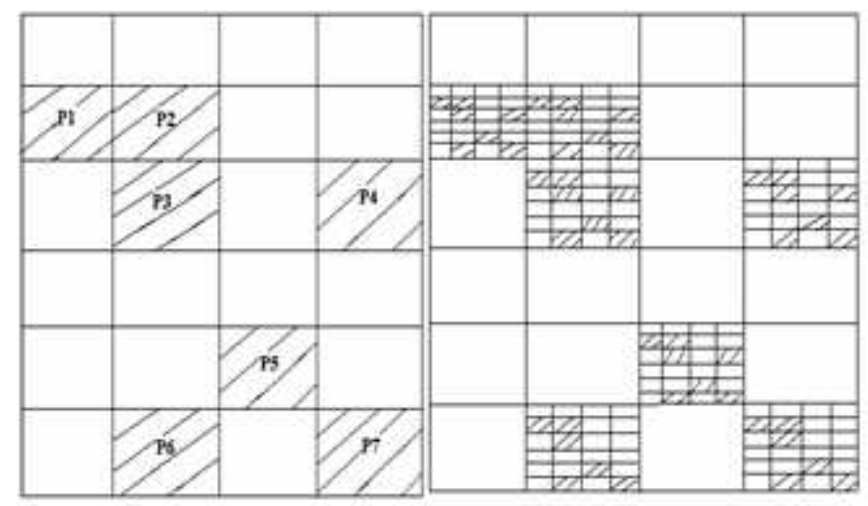

Figure 2: 2D Sierpinski model (step 1 and 2).

For the 3D, we divided the cube into two in $\mathrm{X}$ and $\mathrm{Y}$ directions and four in the $\mathrm{Z}$ direction, then in the boxes of coordinates $\mathrm{I}$, we affected a corresponding probability $\mathrm{P}$ :

$\mathrm{I}=\{(0,0,0),(1,0,0),(1,0,1),(0,1,1),(1,0,2),(1,0,3),(1,1,3)\}$.

$\mathrm{P}=(1 / 20,1 / 15,1 / 5,1 / 10,1 / 5,1 / 10,17 / 60)$

It would be too long to detail the calculation of theoretical multifractal spectrum of this model: we will limit our presentation to express the theoretical $\tau(\mathrm{q})$ function, which define the multifractal spectrum of a probability [8]. However, its possible to take various probabilities to generate others models, for example $\mathrm{p}_{1}=1 / 3, \mathrm{p}_{2}=1 / 4, \mathrm{p}_{3}=0$ and $\mathrm{p}_{4}=5 / 12$. Since the measure is a Bernoulli probability, it comes for the $\tau$ formula:

$$
\tau(q)=\frac{1}{\log (2)} \log \left(\left(\frac{3}{20}\right)^{q} 2^{\beta(q)}+\left(\frac{17}{20}\right)^{q} 2^{\gamma(q)}\right) .
$$

With $\beta$ and $\gamma$ are 2 functions defined as:

$$
\begin{gathered}
\beta(q)=\frac{1}{\log (2)} \log \left(\left(\frac{1}{3}\right)^{q}+\left(\frac{2}{3}\right)^{q}\right) . \\
\gamma(q)=\frac{1}{\log (2)} \log \left(\left(\frac{1}{3}\right)^{q} 2^{\omega(q)}+\left(\frac{2}{3}\right)^{q}\right) .
\end{gathered}
$$

Where,

$$
\omega(q)=\frac{1}{\log (4)} \log \left(\left(\frac{3}{17}\right)^{q}+\left(\frac{2}{17}\right)^{q}+2\left(\frac{6}{17}\right)^{q}\right) .
$$

We deduce the function $\mathrm{f}(\mathrm{q})$ as:

$$
\alpha(q)=\frac{d \tau(q)}{d q} \quad \text { and } \quad f(q)=q \alpha(q)-\tau(q)
$$




\section{Multifractal Spectrum Computing}

We used the method defined by Chhabra and Jensen [5] for multifractal spectrum calculation. We will see below that it requires the definition of the Borel measure. We will bring to this method the implementation of a new measure. The method, for $n$ dimensional signal, is summarized on the flowchart of Figure 6.

After having subdivided the signal into boxes, the $\mu$ measure is defined by the Choquet capacity. In the literature we found many capacities $[12,4]$ with a general definition having the following shape:

$$
\mu_{i}(\delta)=O(x, y)_{B_{i}} g(x, y)
$$

Where $\mathrm{O}$ is an operator dealing with the intensity of pixel $\mathrm{g}(\mathrm{x}, \mathrm{y})$ in the box $\mathrm{i}$ and $\delta$ is the size of the box i. The "sum" operator is used in Chhabra's method, the "max" operator is also widely used. The main drawback of these operators is their lack of sensitivity to the amplitude or to the spatial distribution of the singularities [3].

We defined two new measures for this method. First, we combined one of the previous operators with the gradient " $\Delta$ " computed on each pixel, defined over $n$ axes and the norm [1]. Indeed, we convolved the signal with:

$$
\Delta_{x}=[0,1,-1]
$$

and

$$
\Delta_{y}=\Delta_{x}^{T}
$$

Thus we obtained three measures which are simultaneously sensitive to amplitude and spatial distribution of the singularities. These measures have the following expression:

$$
\begin{gathered}
\mu_{x, i}(\delta)=O \Delta_{x} g(x, y) \\
\mu_{y, i}(\delta)=O \Delta_{y} g(x, y) \\
\mu_{i}(\delta)=\sqrt{\mu_{x, i}(x, y)^{2}+\mu_{y, i}(x, y)^{2}}
\end{gathered}
$$

for each $(\mathrm{x}, \mathrm{y})$ in the box $\mathrm{i}$.

Secondly, a step of the "DBC" method is used to define a measure which we call "DBC-mes", it is defined as follows [4]:

$$
\mu_{i}(\delta)=\frac{p_{i}(\delta)}{\sum_{i} p_{i}(\delta)}
$$

Where $\mathrm{p}_{i}$ is the maximum deviation of gray levels in box $\mathrm{i}$.

Then, we computed the partition function $\tau(\mathrm{q})$, where $\mathrm{q}$ is a real number (Figure 3 ). Finally, we defined the multifractal spectrum by Legendre transform. 


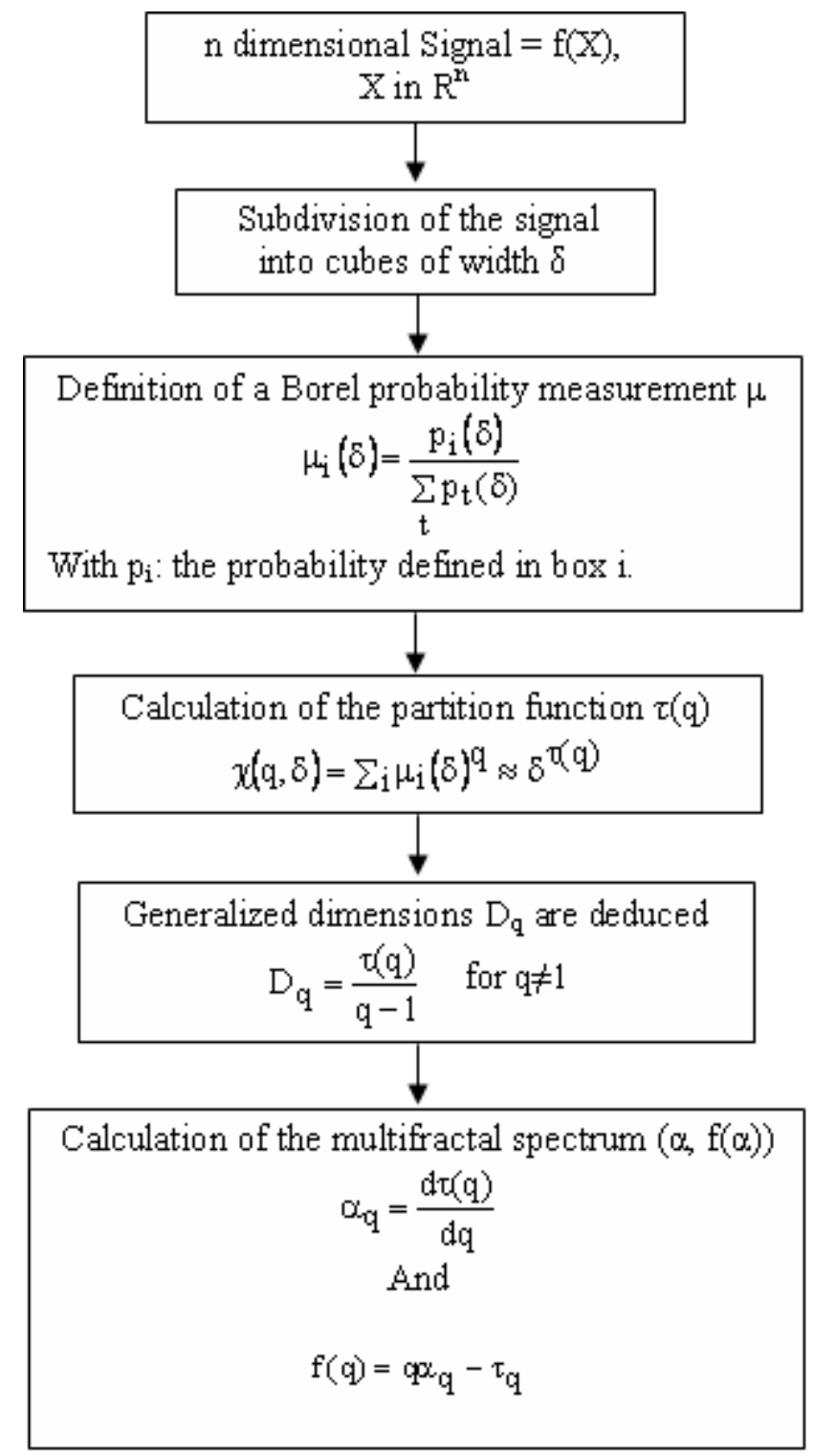

Figure 3: Flowchart of the method. 


\begin{tabular}{|c|c|c|c|}
\hline Measures & $1 \mathrm{D}$ & $2 \mathrm{D}$ & $3 \mathrm{D}$ \\
\hline "Sum" & $1 \times 10^{-8}$ & $1 \times 10^{-8}$ & $1 \times 10^{-8}$ \\
"Max" & $3 \times 10^{-3}$ & $2 \times 10^{-3}$ & $9 \times 10^{-3}$ \\
"Gradient" & $2.5 \times 10^{-2}$ & $2.3 \times 10^{-2}$ & 0.7 \\
"DBC" & $7 \times 10^{-7}$ & $5 \times 10^{-7}$ & $3 \times 10^{-6}$ \\
\hline
\end{tabular}

Table 1: Approximation errors $\sigma$ of the self-similar model between the approximate multifractal spectrum and the theoretical multifractal spectrum. Results are showed for each measure.

\section{Results}

In this paragraph, we compared results between various approximate multifractal spectra and the theoretical multifractal spectrum. So, we compared the representation $(\alpha, \mathrm{f}(\alpha))$. The error of the approximation between representations $\left(\alpha_{1}, \mathrm{f}_{1}\right)$ and $\left(\alpha_{2}, \mathrm{f}_{2}\right)$ is defined by:

$$
\sigma=\sqrt{\left(\sum_{i}\left(\alpha_{1, i}-\alpha_{2, i}\right) / n\right)^{2}+\left(\sum_{i}\left(f_{1, i}-f_{2, i}\right) / n\right)^{2}}
$$

Where $\mathrm{n}$ is the points number.

\subsection{Experimental protocol}

The models algorithms have been developed in C++. We used 6 iterations for the recursive constructions for the self-similar models and 3 iterations for the self-affine model.

For the implementation of the multifractal spectrum calculation, we used the two current measures ("max" and "min") and we compared with the two new measures ("gradient" and "DBC"). We used 91 points to compute these different spectra.

\section{2. $1 \mathrm{D}$ case}

In the Figure 4, we compare Chhabra's method with the analytical solution. We can see that the results are in agreement with the theoretical solution.

We note that in the $1 \mathrm{D}$ case, each measure approximates well the theoretical measure (table 1). Indeed the error $\sigma$ is small for each measure.

\subsection{D case}

We build the model defined in part 3.2, with probabilities $\mathrm{p}_{1}=1 / 5, \mathrm{p}_{2}=1 / 3, \mathrm{p}_{3}=1 / 3, \mathrm{p}_{4}=2 / 15$.

We note that the "gradient" measure gives the worst approximation of the spectrum (table 1 ), however the result remains correct $(\sigma=0.023)$. For the other measures, the results look very satisfactory (Figure 5). Indeed the error $\sigma$ is less than $10 \mathrm{e}-3$ for the three others measures. 


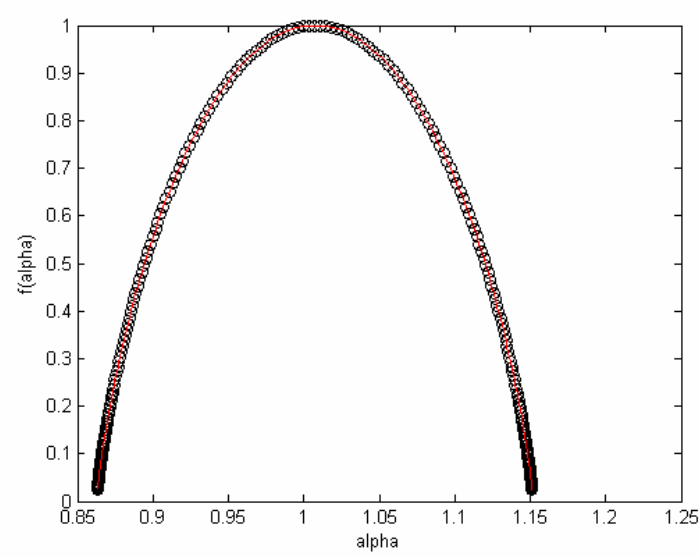

(a) "sum" measure

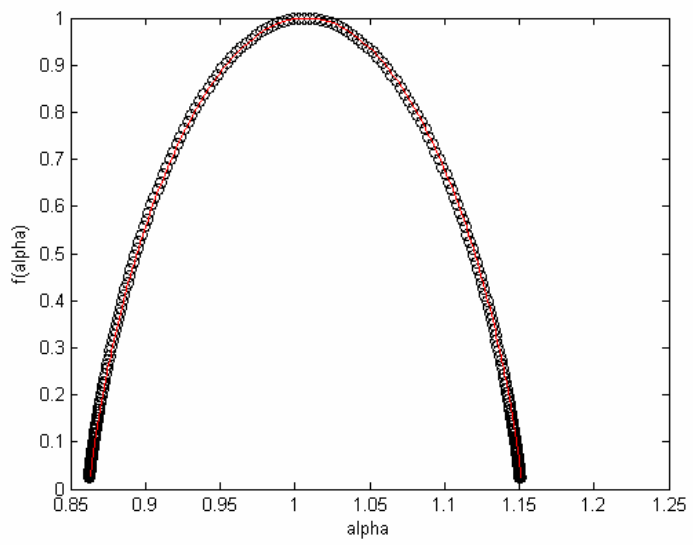

(c) "DBC-mes" measure

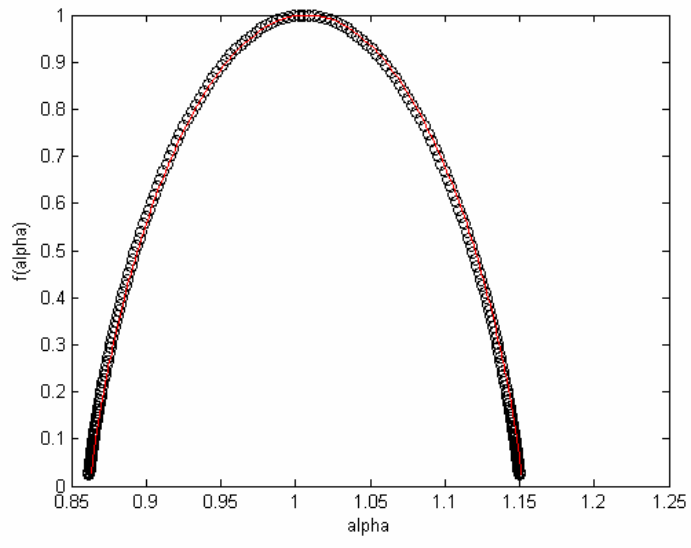

(b) "max" measure

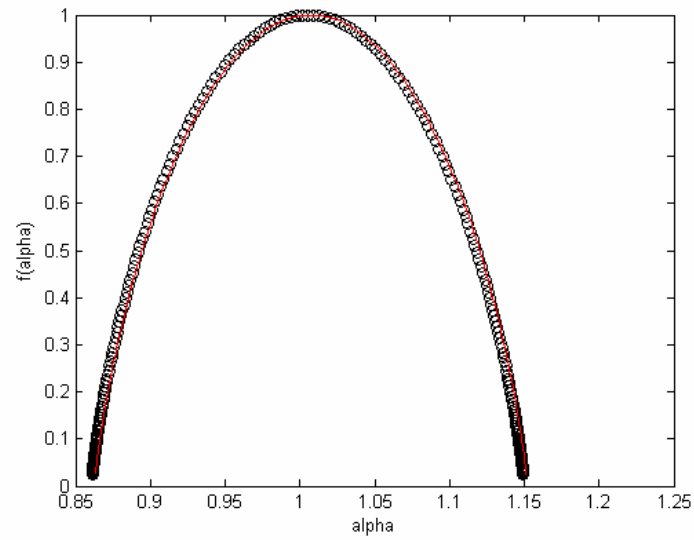

(d) "gradient" measure

Figure 4: Representation of the approximated spectrum and the theoretical spectrum (continuous traced on the figures). 


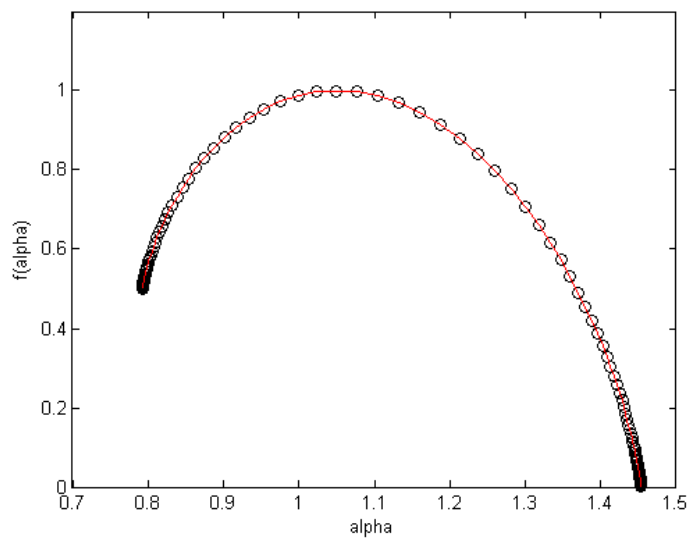

(a) "sum" measure

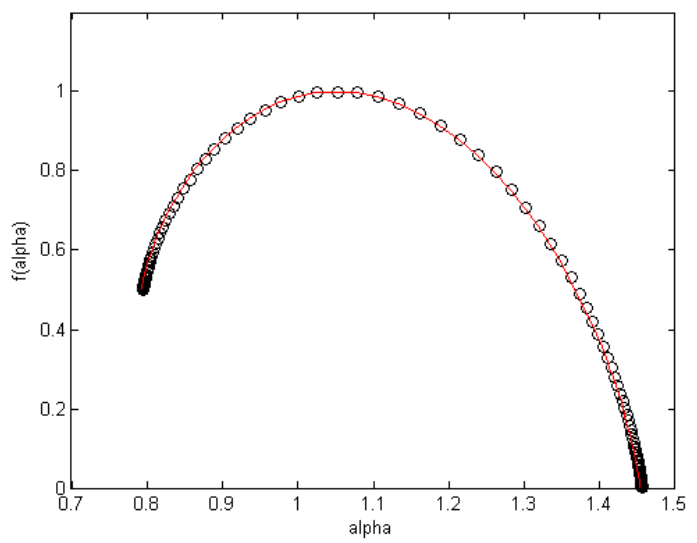

(c) "DBC-mes" measure

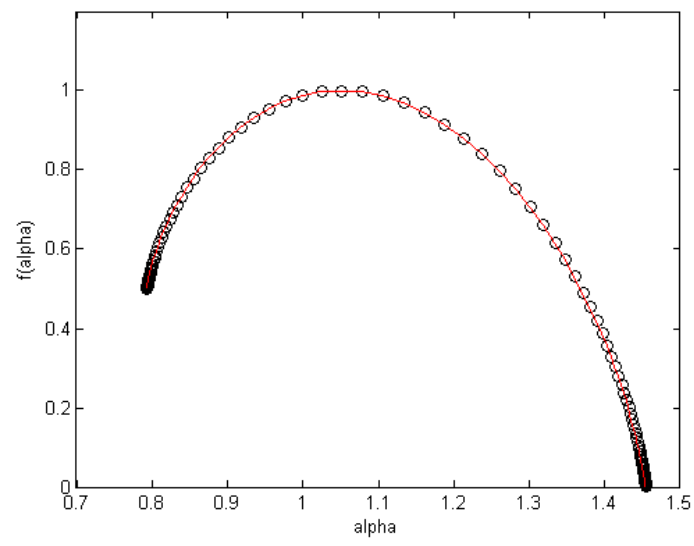

(b) "max" measure

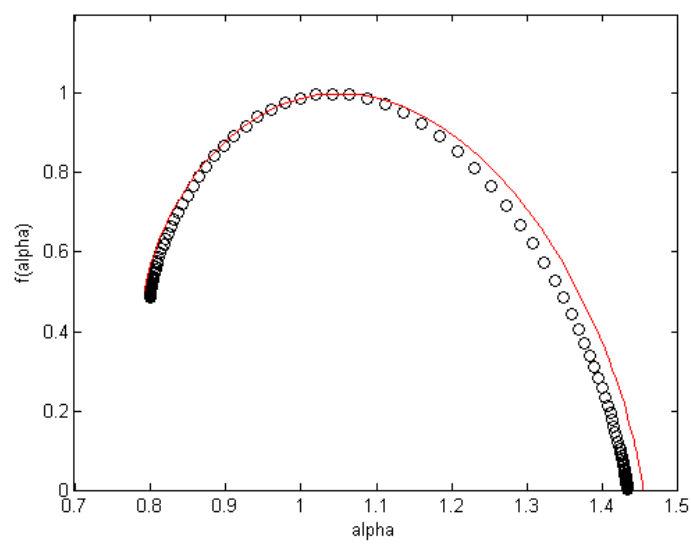

(d) "gradient" measure

Figure 5: Representation of the approximated spectrum and the theoretical spectrum (continuous traced on the figures). 


\subsection{D case}

Concerning the self-similar model, the "gradient" measure is not optimal, whereas the three other measurements approximate the theoretical multifractal spectrum quasi exactly (Figure 6). Indeed for the "gradient" measure, the error is 0.7 (table 1).

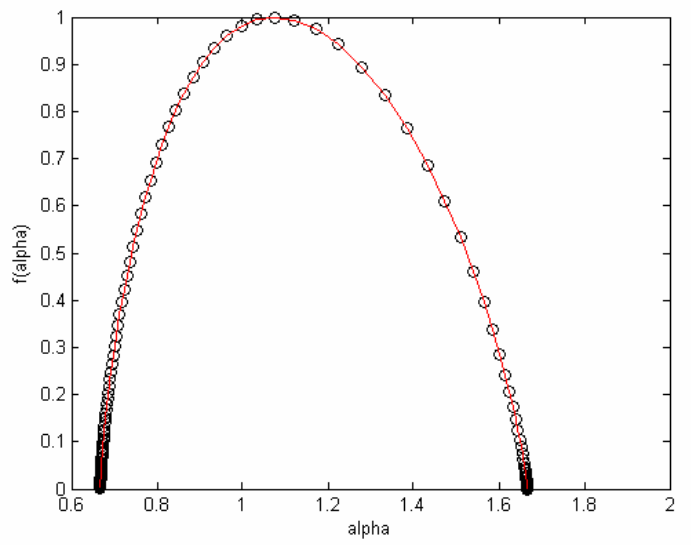

(a) "sum" measure

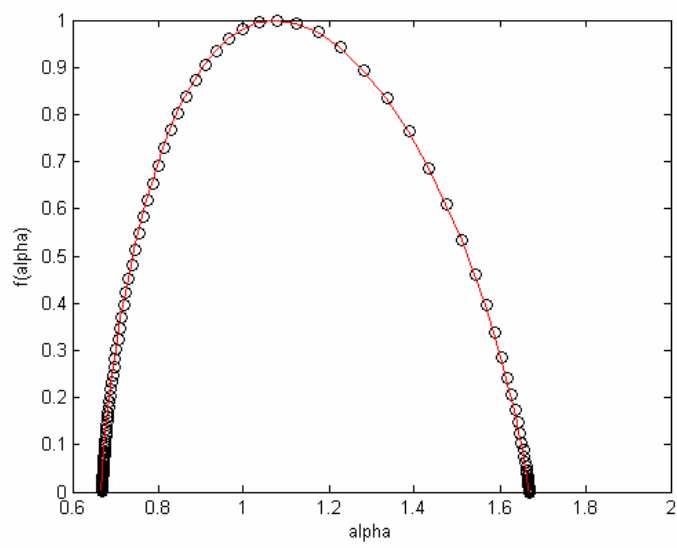

(c) "DBC-mes" measure

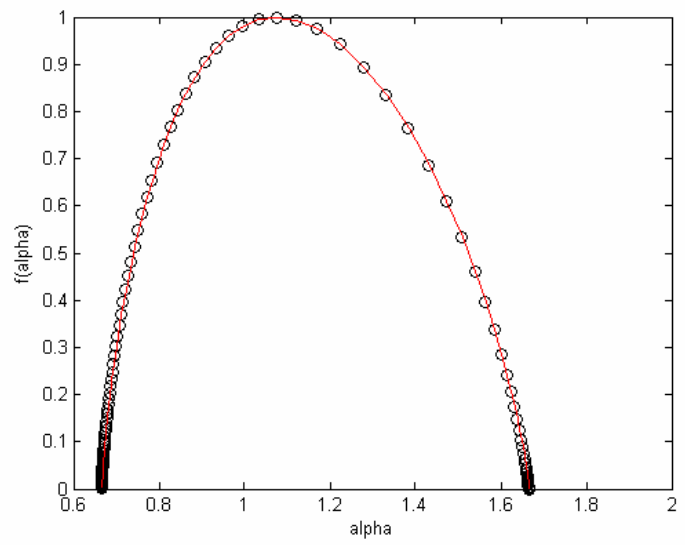

(b) "max" measure

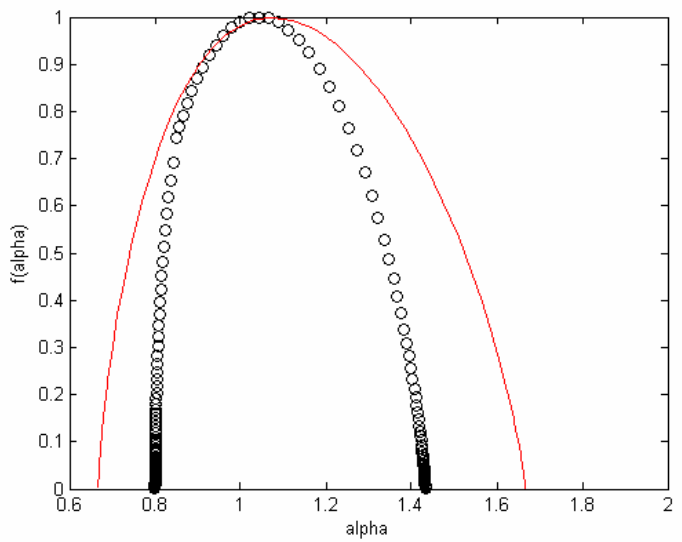

(d) "gradient" measure

Figure 6: Representation of the approximated spectrum and the theoretical spectrum (continuous traced on the figures).

For the self-affine model, the results are completely different. When we apply the "max" and "gradient" measures, results diverge, and we obtain a concave spectrum which is not a good approximation of the model by using "sum" measure. Finally for "DBC-mes" measure, the results are better than the three other ones, the spectrum width $\mathrm{d} \alpha$ is equal to that of the theoretical spectrum (Figure 7). This result is interesting, because the spectrum width can be used like a discriminative value between two populations $[6,17]$. Therefore, it is important to have an exact approximation. 


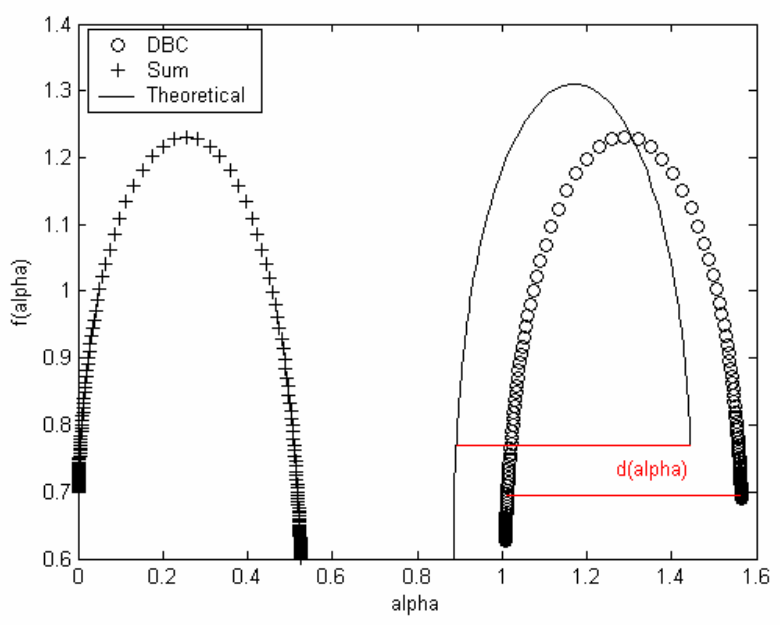

Figure 7: A comparison between calculated multifractal spectra by using the "sum" measurements (left) "DBC-mes" measurements (right), and the theoretical spectrum.

\section{Conclusion}

The aim of this work is to find an absolute value of a multifractal spectrum, that could be used it in other applications than classification (normal/abnormal). For example in the biomedical domain, fractal and multifractal analysis are often used at ends of classification [10,17]. Thereafter we plan to use this analysis for other applications, especially for the characterization of epileptic sources on SPECT images. The second interest is to purpose a theoretical benchmark platform of results confrontation.

Chhabra's method is powerful for self-similar models, but that is less true for non self-similar models. Using "DBC-mes" measure instead of the "sum" (usually used) measure gives the best results. Indeed each measure provides a good approximation for self-similar models, whereas "DBC-mes" measure is the only measure approximating the self-affine model. That's why that will be interesting to add others multifractal models to fill out these results, especially self-affine models.

Finally it would be interesting to test other methods for multifractal spectrum calculation, in particular the algorithms based on the wavelet transform modulus maxima (WTMM) [8] and the wavelet leader [18].

\section{References}

[1] M. Abadi, E. Grandchamp. Texture features and segmentation based on multifractal approach. Iberoamerican Congress on Pattern Recognition, 4225 (2006), 297-305.

[2] A. Arneodo, E. Bacry, F. Argoul, J. Elezgaray, JF. Muzy. Ondelettes, multifractales et turbulence, de l'ADN aux croissances cristallines. Diderot-Science en actes, 1994. 
[3] JP. Berroir, J. Lvy Vhel. Multifractal tools for image processing. In Proc. Scandinavian Confrence on Image Analysis, 1 (1993), 209-216.

[4] B. Chaudhuri, N. Sarkar. Texture segmentation using fractal dimension. IEEE Trans. on Pattern Anal. and Machine Intell., 17 (1995), No. 1, 72-77.

[5] A. Chhabra, RV. Jensen. Direct determination of the f(alpha) singularity spectrum. Phys. Rev. Lett, 62 (1989), No. 12, 1327-1330.

[6] R. Galaska, D akowiec, A. Dudkowska, A. Koprowski, K. Chlebus, J. Wdowczyk-Szulz, A. Rynkiewicz. Comparison of wavelet transform modulus maxima and multifractal detrended fluctuation analysis of heart rate in patients with systolic dysfunction of left ventricle. Annals of Noninvasive Electrocardiology, 13 (2008), No. 2, 155-164.

[7] T. Halsey, M. Jensen, L. Kadanoff, I. Procaccia, B. Shraiman, Fractal measures and their singularities: The characterization of strange sets. Phys. Rev. A, 13 (1986), 1141-1151.

[8] Y. Heurteaux. Estimations de la dimension infrieure et de la dimension suprieure des measures. Ann. Inst. H. Poincar Probab. Statist, 34 (1998), No. 3, 309-338.

[9] S. Jaffard. Multifractal formalism for functions (part I and II). SIAM J. Math. Anal., 24 (1997), 944-970.

[10] P. Kestener, JM. Lina, P. Saint-Jean, A. Arneodo, Wavelet-based multifractal formalism to assist in diagnosis in digitized mammograms. Image Anal. Stereol., 20 (2004), No. 3, 169174.

[11] B. Lashermes, S. Jaffard, P. Abry. Wavelet leader based multifractal analysis. IEEE International Conference on Acoustics, Speech, and Signal Processing, 4 (2005), 161-164.

[12] J. Lvy-Vhel, P. Mignot. Multifractal segmentation of images. Fractals, 2 (1994), No. 3, 371377.

[13] R. Lopes, P. Dubois, AS. Dewalle, M. Steinling, S. Maouche, N. Betrouni. 3D multifractal analysis of cerebral tomoscintigraphy images. Lecture notes in Computer Assisted Radiology and Surgery, 2 (2007), No. 1, 17-18.

[14] BB. Mandelbrot. Fractals and Multifractals : Noise, Turbulence and Galaxies. Vol. 1 of Selecta (Springer Verlag Berlin), 1989.

[15] C. Meneveau, KR. Sreenivasan. Simple multifractal cascade model for fully developed turbulence. Phys. Rev. Lett., 59 (1987), No. 13, 1424.

[16] C. Meneveau, KR. Sreenivasan. The multifractal nature of turbulent energy dissipation. J. Fluid Mech., 224 (1991), 429-484. 
[17] A. Munoz-Diosdado, JL. Del Rio Correa, AF. Brown. Multifractality in time series of human gait. Proceedings of the 25th Annual International Conference of the IEEE., 2 (2003), 17921795.

[18] M. Niu, Q. Liang, G. Yu, F. Wang, Z. Yu. Multifractal analysis of pressure fluctuation signals in an impinging entrained-flow gasifier. Chemical Engineering and Processing, 47 (2008), No. 4, 642-648.

[19] G. Paladin, A. Vulpiani. Anomalous scaling laws in multifractal objects. Phys. Rep., 156 (1987), No. 4, 147-225.

[20] T. Stosic, B. Stosic. Multifractal analysis of human retinal vessels. IEEE Transactions on Medical imaging, 25 (2006), No. 8, 1101-1107.

[21] C. Tricot. Courbes et dimensions fractals, Chap. 20-21, Springer, 1997. 